\title{
TRAIL Spotlight
}

\section{Ionospheric Radio Propagation}

\section{Scott Curtis}

$\mathbf{H}$ ow can our radios receive signals from stations beyond our line of sight? One common mode of transmission and reception of radio waves is "skywave," or ionospheric propagation. In skywave transmission, a radio station transmits medium or high frequency (MF or HF) radio waves away from the Earth and toward the ionosphere, an atmospheric layer extending from approximately 50-400 miles (approximately 80-640 $\mathrm{km}$ ) above the ground. ${ }^{1}$ The ionosphere gets its name from the high concentrations of charged ions and free electrons present at this altitude. These charged particles act as a mirror for the transmitted radio waves, reflecting them back toward Earth. The skywave can bounce once, or many times, between the ionosphere and the Earth, before reception by a radio receiver. We experience skywave reception with long-distance AM radio reception, as well as for shortwave radio and many of the radio bands used by amateur radio "hams."

When Guglielmo Marconi claimed the first successful radio transmission and reception across the Atlantic in 1902, many scientists doubted the achievement, arguing the Earth's curvature prevented such a contact. ${ }^{2}$ The ionosphere was not then known to exist, and the presence of ionized elements in the upper atmosphere was not scientifically established until the 1920s (pp. 6-10). By the 1920s, Marconi advanced radio technology by recording longer distance contacts using higher transmission frequencies (pp. 6-10).

After the Second World War, the United States Department of Commerce, National Bureau of Standards (NBS) published NBS Circular 462, entitled Ionospheric Radio Propagation to respond to a need to "...present the elementary principles of sky-wave or ionospheric radio-wave propagation at high frequencies and their practical application to the problems of radio communication." Ionospheric Radio Propagation helped radio operators, technicians, and lay people who had not studied advanced electrodynamics to understand what happens when radio waves interact with the ionosphere. With this knowledge, technicians, engineers, and radio operators could better use other government information resources like Ionospheric Predictions, produced by the Central Radio Propagation Laboratory of the National Bureau of Standards. ${ }^{4}$ Ionospheric Predictions provided data on predicted ionospheric conditions to allow calculations about the proper frequency to use for a transmission to reach the desired distance.

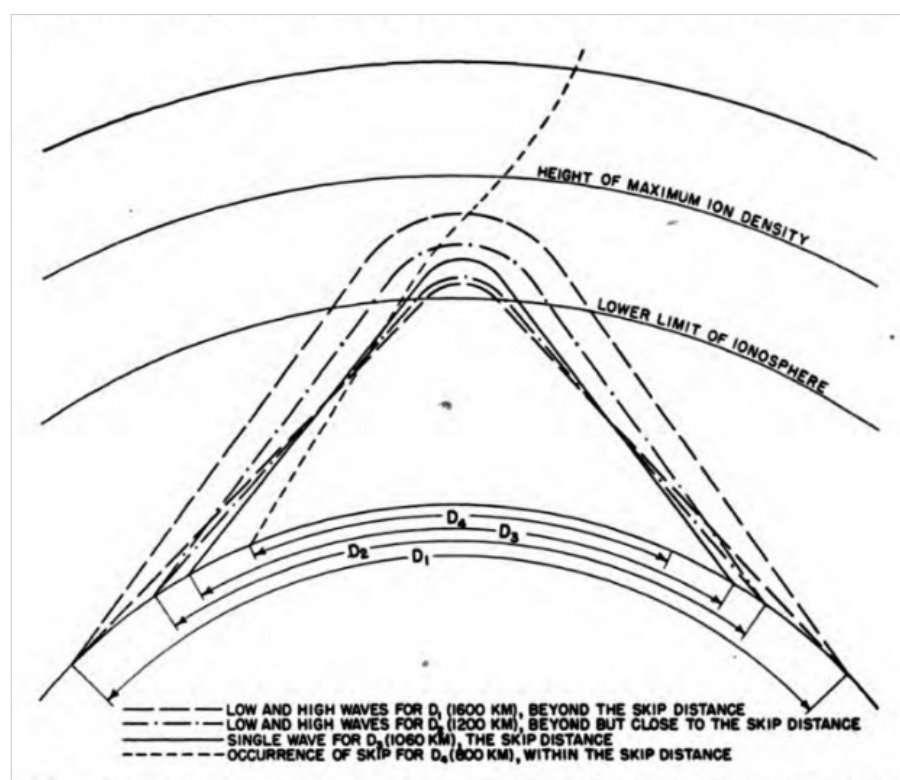

FIGURE 6.5. Fixed frequency-varying distance (curved earth, curved ionosphere assumed).

Diagram illustrating how received distance varies for fixed transmission frequencies for a given angle of reflection off the ionosphere. Central Radio Propagation Laboratory (US), lonospheric Radio Propagation, National Bureau of Standards, Circular 462 (Washington, DC: Government Printing Office, 1948), 71.

Major international scientific collaborations such as The International Geophysical Year (1957-58) and The International Year of the Quiet Sun (1964-65) advanced scientific understanding of the ionosphere and necessitated an update to Ionospheric Radio Propagation. 5 A substantially revised and rewritten book appeared in 1965 as NBS Monograph 80, also entitled Ionospheric Radio Propagation, this time with primary author Kenneth Davies. NBS Monograph 80 has been cited almost 700 times in Google Scholar, and its 1990 revision has been cited nearly 1,800 times.

Find more technical reports using TRAIL, the Technical Report Archive and Image Library, at www.technicalreports.org.

Scott Curtis (curtissa@umkc.edu), Teaching \& Learning Librarian, University of Missouri, Kansas City 


\section{TRAIL Spotlight}

\section{Notes}

1. "Ionosphere or Thermosphere," in Funk \& Wagnalls New World Encyclopedia, 2018.

2. J. Bach Andersen, "History of Communications/Radio Wave Propagation from Marconi to MIMO," IEEE Communications Magazine 55, no. 2 (February 2017): 6-10, https://doi.org/10.1109/MCOM.2017.7841460.

3. Central Radio Propagation Laboratory (US), Ionospheric Radio Propagation, National Bureau of Standards, Circular 462 (Washington, DC: Government Printing Office, 1948), http://www.technicalreports.org/trail/detail/15727/. 